NASA/TM= / qij - 208123

$$
\begin{gathered}
\text { M-OS-TM } \\
M X \| 1
\end{gathered}
$$

\title{
INFLUENCE OF IMPACTOR MASS ON THE DAMAGE CHARACTERISTICS AND FAILURE STRENGTH OF LAMINATED COMPOSITE PLATES
}

Damodar R. Ambur and Heather L. Kemmerly

NASA Langley Research Center

Hampton, VA

Presented at the 39th AIAA/ASME/ASCE/AHS/ASC Structures, Structural Dynamics, and Materials Conference

AIAA Paper No. 98-1784

\author{
Long Beach, California
}

April 20-23, 1998 
- 
AIAA 98-1784

\title{
INFLUENCE OF IMPACTOR MASS ON THE DAMAGE CHARACTERISTICS AND FAILURE STRENGTH OF LAMINATED COMPOSITE PLATES
}

\author{
Damodar R. Ambur* and Heather L. Kemmerly ${ }^{\dagger}$ \\ NASA Langley Research Center \\ Hampron, VA
}

\begin{abstract}
$\underline{\text { Abstract }}$
The results of an experimental study of the effect of impactor mass on the low-speed impact response of laminated flat composite plates is presented. Droppedweight impact response, damage characteristics, and residual strengths of quasi-isotropic flat plates are presented for a range of energy levels by systematically varying the mass of the impactor. Measured contact forces and damage areas are also presented. The results indicate that the contact force and damage area are nonlinear functions of the impactor mass and vary considerably over the entire range of energy levels considered. The different damage levels induced in a plate specimen when impacted at a given energy level with impactors of different masses significantly influence its compressive residual strength. The results provide clear and consistent trends in contact force, damage area, and compression-after-impact strength when the data are expressed as a function of the impactor momentum.
\end{abstract}

\section{Introduction}

The effect of low-speed impact damage on the compression strength of laminated composite structures has been studied extensively by many researchers over the past several years. Test data show that the compression strength of composite structures can be significantly reduced by low-speed impact damage, even if the damage is not detectable by visual inspection. Many of the studies of the effects of low-speed impact damage reported in the literature have focused on the influence of impactor mass on the low-speed impact

\footnotetext{
* Assistant Head, Structural Mechanics Branch. Associate Fellow, AIAA.

† Aerospace Engineer, Structural Mechanics Branch.

Copyright (1) 1998 by the American Institute of Aeronautics and Astronautics, INC. No copyright is asserted in the United States under Title 17, U.S. Code. The U.S. Government has a royalty-free license to exercise all rights under the copyright claimed herein for government purposes. All other rights are reserved by the copyright owner.
}

response of composite plates. Studies reported in Refs. 1-6 address the effects of impactor mass on the impact contact forces and damage areas that result from lowspeed impact. Ref. 1 uses spherical steel projectiles that weigh $0.0022 \mathrm{lb}$ and $0.00132 \mathrm{lb}$ and also a nylon sphere that weighs $0.000374 \mathrm{lb}$ to study the effects of impactor mass and impactor material on the damage initiation threshold. In this study (Ref. 1) it was shown that the energy needed to initiate damage increases with an increase in the impactor mass. Refs. 2-5 investigated the effects of low-speed and high-speed impacts where impactors of different masses were used. Ref. 2 and 3 studied perforation threshold energy levels using impator weights of $1.5 \mathrm{lb}$ for low-speed impacts and $0.0022 \mathrm{lb}$ for high-speed impacts. The studies in Refs. 4-5 discussed the dynamic effects associated with using diferent impactor weights. Ref. 5 concluded that for a given impact energy when the impactor velocity is increased, the damage area decreases and the residual strength of the plate increases. A similar study in Ref. 6 suggested that the dynamics of the impact event is a function of the mass of the plate and the impactor and concluded that impact with a low mass at high speed results in greater forces on the plate when compared to an impact with a high mass at low speed. Most of these efforts address impacts due to a projectile (small mass propelled at a high speed), but not due to a dropped-weight impactor.

Dropped-weight impact tests are performed by researchers using different weights; these weights range typically from $2.5 \mathrm{lbs}$ to $25 \mathrm{lbs}$. The general practice for obtaining the compression-after-impact (CAI) test data for a composite material has been to perform a compression test on a flat plate which is impact damaged at its center with a dropped-weight impactor of a given mass. The information generated using this method is then used to design wing and fuselage structures for impact damage tolerance. The maximum contact force results for simply supported (45/0/$45 / 90)_{2 s}$ quasi-isotropic plates of different radii subjected to a central impact are presented in Fig. 1. These specimens were made from Hercules, Inc. AS4 graphite 
fibers preimpregnated with Hercules, Inc. 3502 epoxy resin. The typical properties for this material system are provided in Table 1 . The contact force results in Fig. 1 are generated using the analysis method presented in Ref. 7 for an impact-energy level of $7 \mathrm{ft}$-lbs with different combinations for impactor mass and impactor speed. In this figure, which is plotted with impactor speed on the abscissa, increase in the impactor speed suggests a decrease in the impactor mass for a given impact-energy level. These results clearly indicate that impactor weights of less than $0.25 \mathrm{lb}$ (corresponding to impactor speeds of less than $578 \mathrm{in} / \mathrm{sec}$ ) influence the magnitude of the maximum contact force for plates which have large curvature. Similar results for flat sandwich plates with $(45 / 0 /-45 / 90)_{2 s}$ quasi-isotropic AS4/3502 graphite-epoxy facesheets are presented in Fig. 2 for different core stiffnesses. The analysis approach presented in Ref. 8 has been used to generate these results. For the range of core densities used in this limited study, it appears that the impactor mass values of less than $0.25 \mathrm{lb}$ have an insifgnificant effect on the contact force results. This insensitivity to impactor mass is due to the very local nature of the impact response for facesheets supported on their entire surface area. Therefore, there is a need to study the influence of impactor mass on the impact response, damage characteristics and compression-after-impact strength of composite flat plates. There is very limited information in the literature that relates the residual strength of composite flat plates subjected to low-speed impact damage with different masses over a range of impact-energy levels.

An analytical effort to identify the influence of impactor mass on the contact force and surface strains of $(45 / 0 /-45 / 90)_{6 s}$ quasi-isotropic flat plates was reported in Ref. 9. It was identified in this work that for a given impact energy level, the effect of increasing the impactor mass results in a marginal increase in the maximum contact force and surface strain values. This result is consistent with the analytical maximum contact force data presented in Fig. 3 for this 48-plythick flat plate. The contact force due to an impact event is a major factor in the resulting damage and the residual strength of the plate, it is important to gain an understanding of the influence of the impactor mass on the plate response. A normalized impactor momentum approach was also suggested in Ref. 9 for relating the analytical plate contact force and strain results for impacts with different impactor weights.

The present paper will present the results of an experimental study of the influence of impactor mass on the damage characteristics and compression residual strength of 48-ply, quasi-isotropic, laminated composite flat plates. The experimental contact force results, the damage area results obtained by using a C-scan procedure, and the compression residual strength results after impact for different impact energy levels obtained by varying the impactor masses are summarized, and the dependence of these parameters on impactor mass is identified. The potential for using impactor momentum as a parameter to identify clearly the trends in plate impact response results is discussed.

\section{Test Specimens and Methods}

The 48-ply-thick, $(45 / 0 /-45 / 90)_{6 s}$ quasi-isotropic flat plate test specimens used in this experimental study were fabricated from Hercules, Inc. AS4 graphite fibers preimpregnated with Hercules, Inc. 3502 epoxy resin. A dropped-weight impact test machine described in Reference 9 was used for centrally impacting the specimens at energy levels ranging from $7.5 \mathrm{ft}-1 \mathrm{bs}$ to 25 ft-lbs with impactor weights ranging from 2.5 to 20.0 lbs. The test matrix used for this study is presented in Table 2. As shown in this table each impactor weight has been used to impact plate specimens at five different impact energy levels. The instrumented dropped-weight impactor with a 0.5 -in.-diameter hemispherical tip was dropped from different heights to generate different impact-energy levels. The contact force data during the entire impact event were gathered by means of a load cell located in the impactor. The contact force data was recorded using a NEFF 495 high-speed data acquisition system. The plates had unsupported dimensions of 9in. length and 5-in. width and all plate edges were simply supported. After impacting the specimens, an ultrasonic C-scan procedure was used to determine the impact damage areas for the specimens. The specimens were instrumented with electrical resistance strain gages and the compression residual strength of the specimens was determined by loading each damaged specimen to failure in a 300-kip capacity hydraulic test machine.

\section{$\underline{\text { Results and Discussion }}$}

The effects of increasing impactor mass on the contact force results are presented in Fig. 4. In this figure the maximum contact force results corresponding to impacts with increasing energy levels are plotted for different impactor weights. All the data sets exhibit a nonlinear trend between the impact energy levels and the contact force. For impactor weights of $2.5 \mathrm{lbs}$ and 5.0 lbs, the maximum contact force magnitude increases as the impactor weight increases. This response is due to damage initiation in the plate as the impact-energy level is increased. The initiation of damage results in a decreased value for the maximum contact force that is 
developed due to impact. It has been shown in Reference 10 that, with an impactor weight of $5.0 \mathrm{lbs}$, damage initiates at approximately $12 \mathrm{ft}-\mathrm{lbs}$ in this plate specimen. The contact force magnitudes for impacts with 10.0 and $20.0 \mathrm{lb}$ weights indicate a trend which suggests that the contact force magnitudes for impacts using these weights are smaller than that for the 2.5 and $5.0 \mathrm{lb}$ impactor weights. The contact force results for both the 10.0 and $20.0 \mathrm{lb}$ impactors also exhibit an increasing and then a decreasing trend; with the curves shifted along the abscissa as the impactor weight is increased. If a decrease in the positive slope of the contact force results is an indication of damage initiation in the plate, these results suggest that the impact-damage-initiation threshold energy levels decrease and then increase as the impactor mass is increased. These results have no consistently general trend and it appears that the plate response, when impacted with the $10.0 \mathrm{lb}$ and $20.0 \mathrm{lb}$ weights, is affected significantly by the dynamic interaction of the plate and the impactor mass resulting in a plate global bending response.

The damage area results from the $\mathrm{C}$-scan are presented in Fig. 5 for increasing energy levels and impactor masses. It is to be understood here that the damage areas calculated using $C$-scan images are qualitative indications of damage and cannot be used to interpret directly the compressive residual strength of the plate. Similar to the trends observed for the contact force results, when the impactor weights are $2.5 \mathrm{lbs}$ and $5.0 \mathrm{lbs}$, the resulting damage areas increase with decreasing weight with the larger impactor mass causing more damage. When impacted with impactor weights of $10.0 \mathrm{lbs}$ and $20.0 \mathrm{lbs}$, larger damage area seems to develop as the impact-energy levels are increased. For impacts from the $10.0 \mathrm{lb}$ and $20.0 \mathrm{lb}$ weights, the damage areas for energy levels below $15 \mathrm{ft}$ lbs are comparable to the damage areas for impacts from impactor weights of $2.5 \mathrm{lbs}$ and $5.0 \mathrm{lbs}$ for the corresponding energy levels. For values of impact energy exceeding $15 \mathrm{ft}$-lbs, the resulting damage area gradually increases with increasing energy. A change in the dynamic interaction of the test specimen and the impactor results in a change in the test specimen impact damage mechanisms as the impactor mass is increased.

The compression-after-impact (CAI) test results for the test specimens are plotted in Fig. 6 as a function of the impact-energy levels generated using different impactor weights. These results indicate a generally decreasing trend for specimen failure loads as the impactor energy is increased. There is an insignificant difference in compression failure loads for specimens impacted at an energy level of approximately $15 \mathrm{ft}-\mathrm{lb}$ or less. For impact energy levels greater than $15 \mathrm{ft}-\mathrm{lbs}$ this variation increases and reaches a maximum value of approximately 20 percent for $25 \mathrm{ft}$-lb impact energy. Plate specimens impacted at a given energy level using larger impactor weights appear to have a higher CAI strength. Although this result is consistent with the contact force and damage area results presented previously, these data do not have a consistent trend over the entire range of energy levels considered in this study.

The same impact test data are presented in Figs. 7-9 with impactor momentum as a parameter to help identify trends in the results. The contact force, impact damage area and the CAI strength results are shown to be nonlinear functions of the impactor momentum. The nonlinear trend for the maximum contact force results is not due to large plate deformations (Ref. 11) during the impact event but is a consequence of the changes in stiffness of the plate at the impact location. These results exhibit trends which can be used to identify the effect of momentum on the plate impact response. From this presentation of the data, it can be seen that for two weights impacting with a specified momentum, the heavier impactor results in a smaller impact damage area. This conclusion, with the results visualized in this manner, are consistent with the findings in Ref. 1 where increasing the projectile mass is shown to result in a decrease in the impact damage area. Similarly, the CAI strength data suggest that, for a given impactor momentum, the lighter impactor results in a lower compression failure load. Scaling of the contact force results based on ratios of impactor momentum as described in Ref. 9 and using a similar approach for damage area results is not applicable to the set of results involving impact energies which result in damage to the plates. Contact force and damage area results are nonlinear functions of impact energies for these impact-enrgy levels.

\section{Concluding Remarks}

An experimental study has been conducted to study the effects of dropped-weight impactor mass on the impact response and compression residual strength of laminated composite flat plates. The impact response parameters studied are the contact force obtained from the instrumented dropped weight and the damage area obtained form ultrasound C-scan images. The experimental results suggest that as the impact energy increases, the contact force values for all the impactor masses considered in this study. The reductions in contact force as the impact energy increases is due to damage initiation and a change of damage modes in the specimens. As the mass of the impactor is increased, 
the contact force data shifts to the right along the axis with increasing energy levels. This trend suggests that the damage area decreases as the mass of the impactor is increased. The damage area results thus follow a trend that is consistent with the contact force results. The compression-after-impact test results indicate that the compression strength of a plate varies when impactors of different weights are used to impact the plate at a given impact-energy level. This variation in results is determined to be 10 to 20 percent over the impact energy range investigated in this study.

Scaling of the contact force, damage area and compression failure strength results with impactor momentum (as suggested in Ref. 9) as a parameter may not be used since the results are a nonlinear function of the impactor momentum. The nonlinearities occur when the impact energies are greater than those required for damage initiation in the plate specimen. Presenting the test results as a function of the impactor momentum, however, appears to provide clear trends for plate response.

\section{References}

1. Cantwell, W. J. and Morton, J., "The Influence of Varying Projectile Mass on the Impact Response of CFRP," Composite Structures, Vol. 13, 1989, pp. 101-114.

2. Cantwell, W. J. and Morton, J., "Comparison of the Low and High Velocity Impact Response of CFRP," Composites, Vol. 20, No. 6, 1989, pp. 545.

3. Delfosse, D., Poursartip, A., Coxon, B. R., and Dost, E. F., "Non-Penetrating Impact Behavior of CFRP at Low and Intermediate Velocities," Composite Materials: Fatigue and Fracture - Fifth Volume, ASTM
STP 1230, R. H. Martin, Ed., American Society for Testing and Materials, Philadelphia, 1995, pp. 333-350. 4. Christoforou, A. P., and Swanson, S. R., "Analysis of Simply-Supported Orthotropic Cylindrical Shells Subject to Lateral Impact Loads," Journal of Applied Mechanics, Vol. 57, June 1990, pp. 376.

5. Ghaseminejhad, M. N., Parvizi-Majidi, A., "Impact Behaviour and Damage Tolerance of Woven Carbon Fibre-Reinforced Thermoplastic Composites," Composites, Vol. 21, No. 2, March 1990, pp. 155.

6. Cairns, D. S., and Lagace, P. A., "Transient Response of Graphite/Epoxy and Kevlar/Epoxy Laminates Subjected to Impact," AIAA Journal, Vol. 27, No. 11, November 1989, pp. 1590.

7. Ambur, D. R., and Starnes, J. H., "Nonlinear Response and Damage Initiation Characteristics of Composite Curved Plates Subjected to Low-Speed Impact," AIAA 97-1343-CP, April 1997.

8. Ambur, D. R., and Cruz, J. R., "Low-Speed Impact Response Characteristics of Composite Sandwich Panels," AIAA 95-1460-CP, April 1995.

9. Prasad, C. B., Ambur, D. R., and Stames, J. H., "Response of Laminated Composite Plates to LowSpeed Impact by Different Impactors," AIAA Journal, Vol. 32, No. 6, June 1994, pp. 1270.

10. Ambur, D. R., Starnes., J. H., and Prasad, C. B., "Low-Speed Impact Damage-Initiation Characteristics of Selected Laminated Composite Plates," AIAA Journal, Vol. 33, No. 10, October 1995, pp. 1919-1925.

11. Ambur, D. R., Starnes., J. H., and Prasad, C. B., "Influence of Transverse Shear and Large Deformation Effects on the Low-Speed Impact Response of Laminated Composite Plates," NASA TM-107753, Aprill 1993.

Table 1. Mechanical properties of AS4-3502 graphite-epoxy unidirectional tape material.

$\begin{array}{lr}\text { Longitudinal modulus, } \mathrm{E}_{1}(\mathrm{Msi}) & 20.00 \\ \text { Transverse modulus, } \mathrm{E}_{2}(\mathrm{Msi}) & 1.30 \\ \text { In-plane shear modulus, } \mathrm{G}_{12}(\mathrm{Msi}) & 0.87 \\ \text { Transverse shear modulus, } \mathrm{G}_{23}(\mathrm{Msi}) & 0.51 \\ \text { Transverse shear modulus, } \mathrm{G}_{13}(\mathrm{Msi}) & 0.87 \\ \text { Major Poisson's ratio, } \boldsymbol{v}_{12} & 0.30\end{array}$


Table 2. Impactor weight and energy level combinations for impact tests.

Impactor weight, lb Impact energy, ft-lb

\begin{tabular}{ccccccc}
2.5 & 7.5 & 10.0 & 12.5 & 15.0 & 20.0 & \\
5.0 & - & 10.0 & 12.5 & 15.0 & 20.0 & 25.0 \\
10.0 & - & 10.0 & 12.5 & 15.0 & 20.0 & 25.0 \\
20.0 & - & 10.0 & 12.5 & 15.0 & 20.0 & 25.0 \\
\hline
\end{tabular}

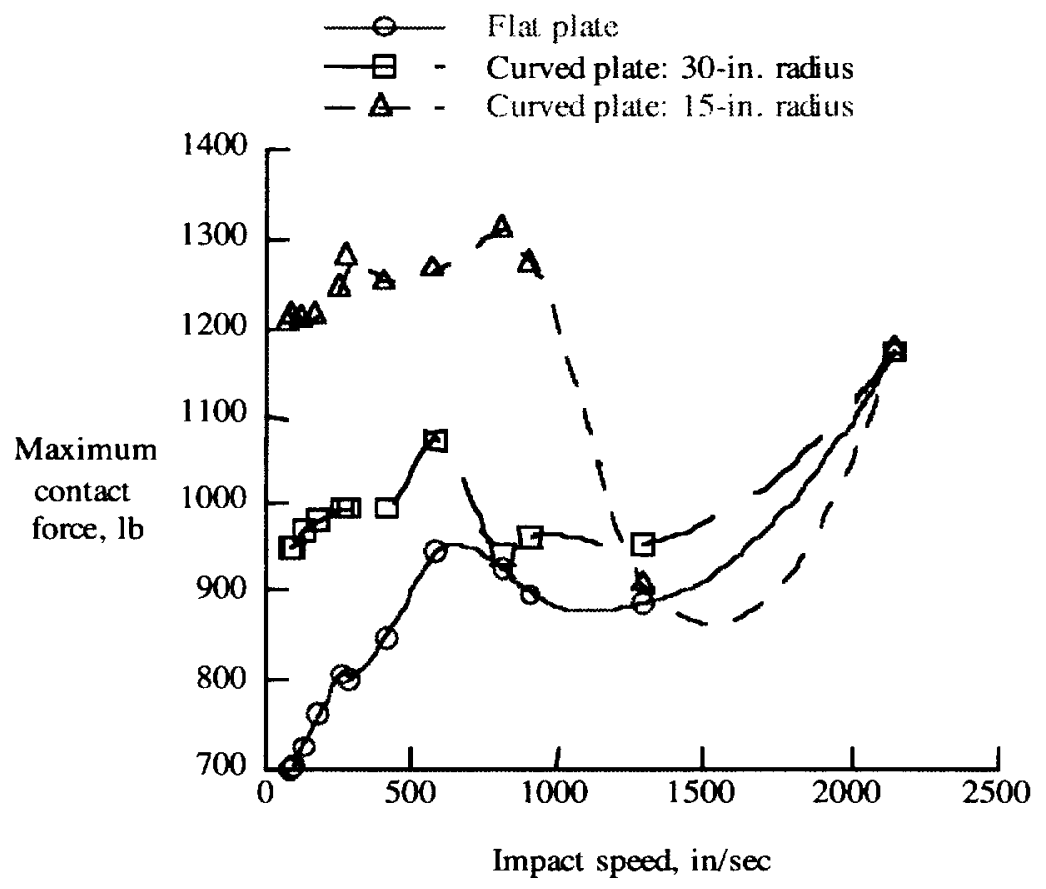

Figure 1. Influence of impactor weight on the contact force results for $(45 / 0 /-45 / 90)_{2 s}$ plates impacted with an energy level of $7 \mathrm{ft}-\mathrm{lbs}$. 


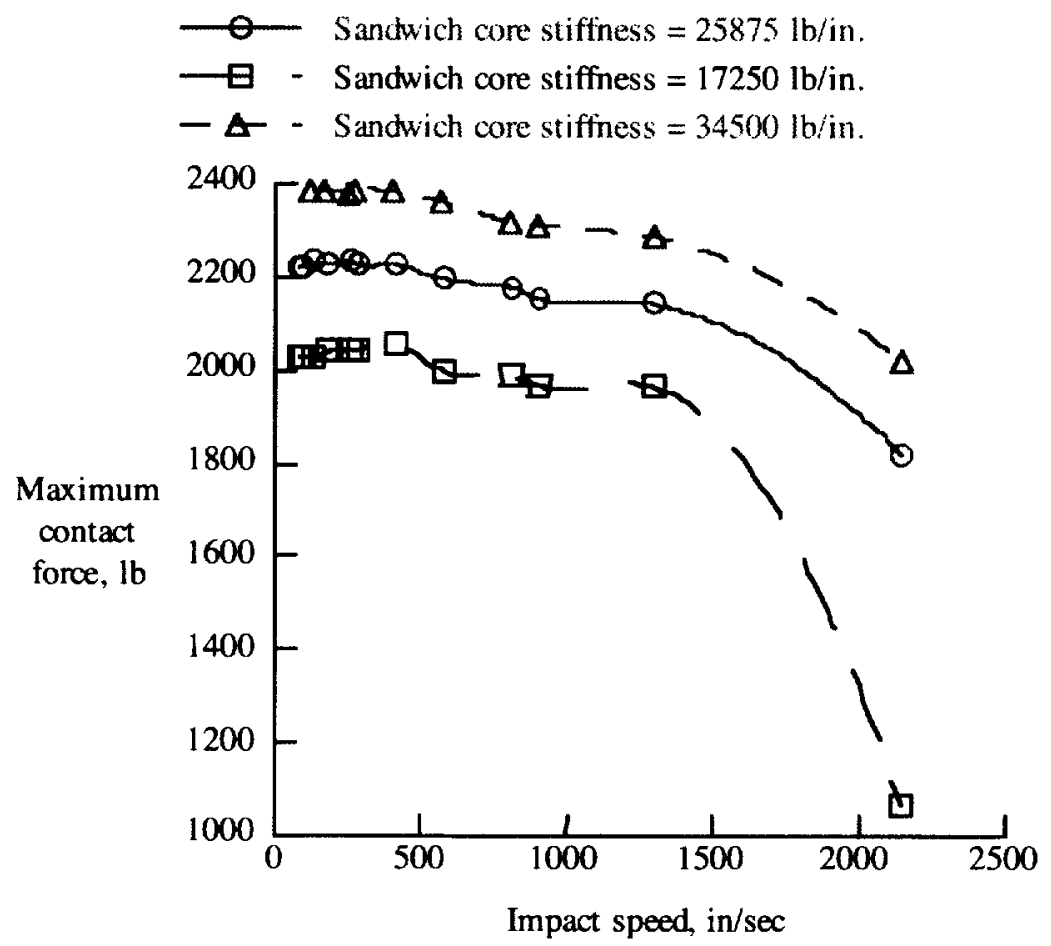

Figure 2. Influence of impactor weight on the contact force results for sandwich plates made of $(45 / 0 /-45 / 90)_{2 \mathrm{~s}}$ facesheets with core of different stiffnesses and impacted with an energy level of $7 \mathrm{ft}$-lbs.

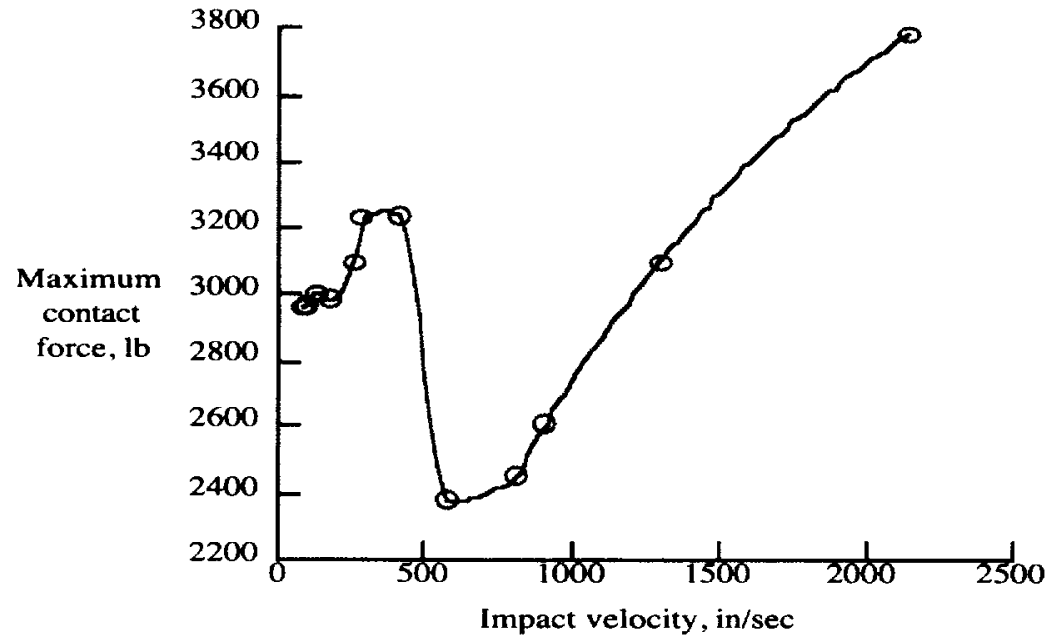

Figure 3. Influence of impactor weight on the contact force results for $(45 / 0 /-45 / 90)_{6 \mathrm{~s}}$ flat plate impacted with an energy level of $9 \mathrm{ft}$-lbs. 


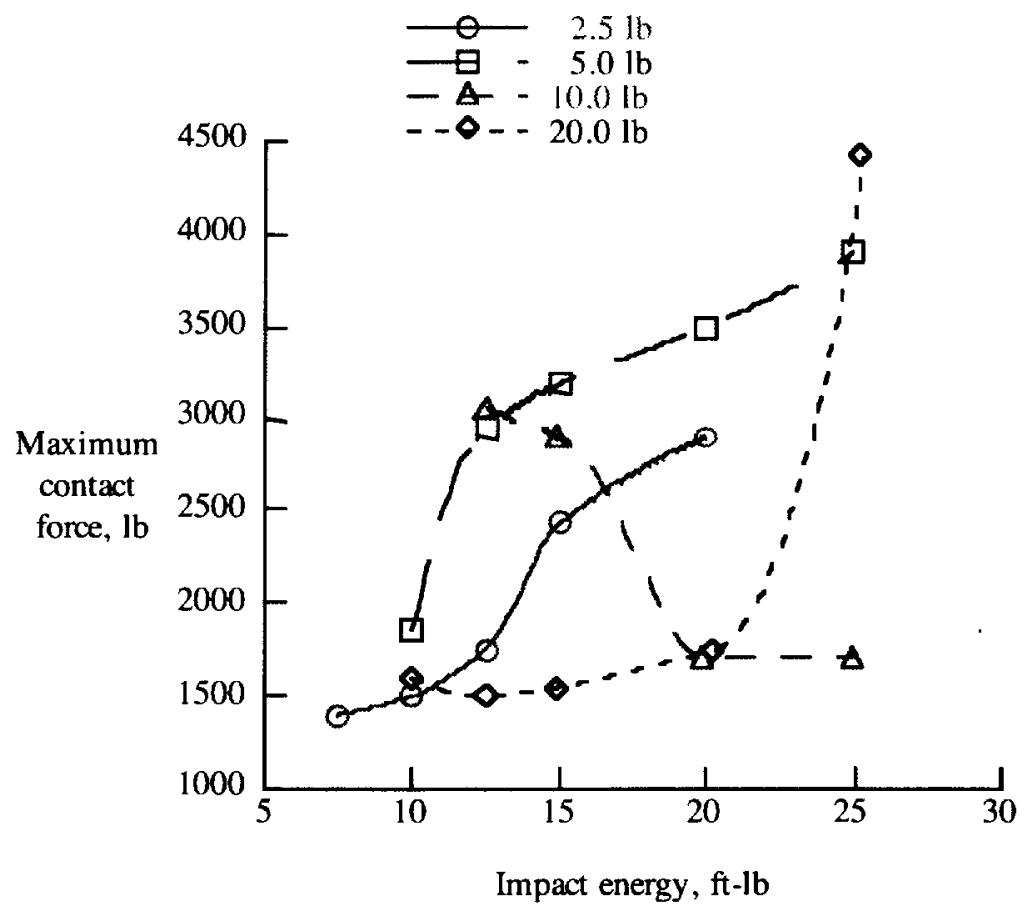

Figure 4. Experimental contact force results for impacts with increasing energy levels and with different impactor weights.

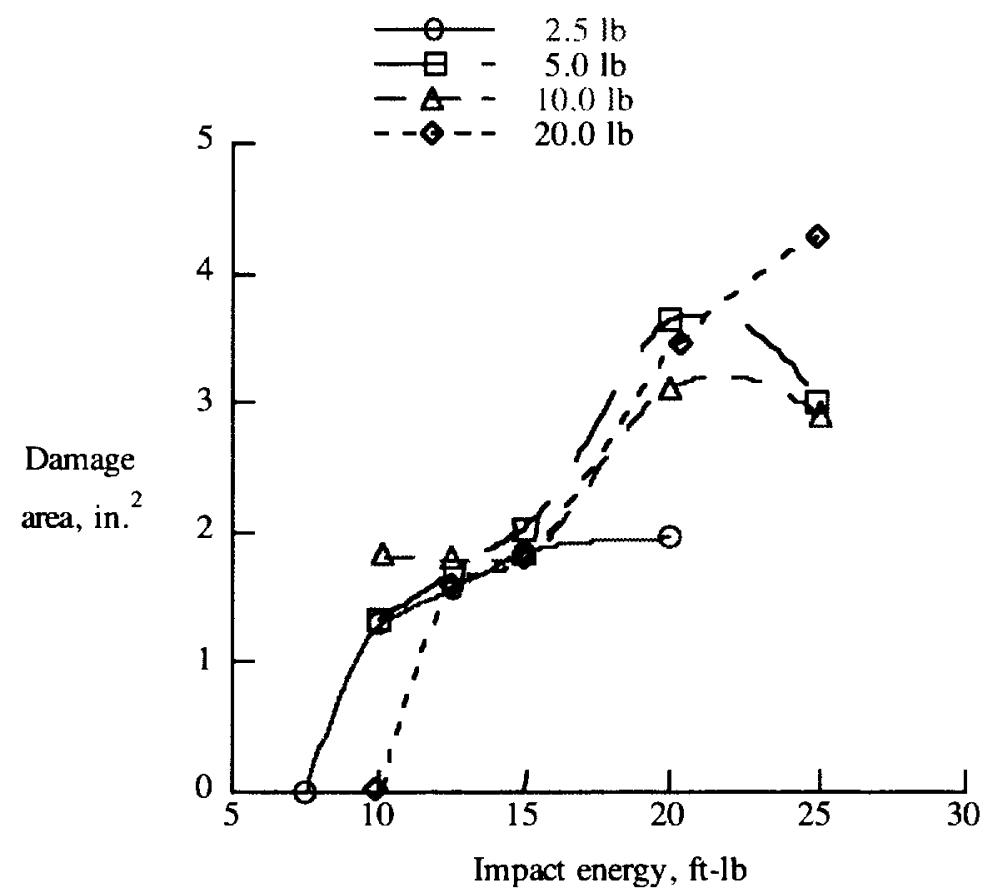

Figure 5. Experimental damage area results for impacts with increasing energy levels and with different impactor weights. 


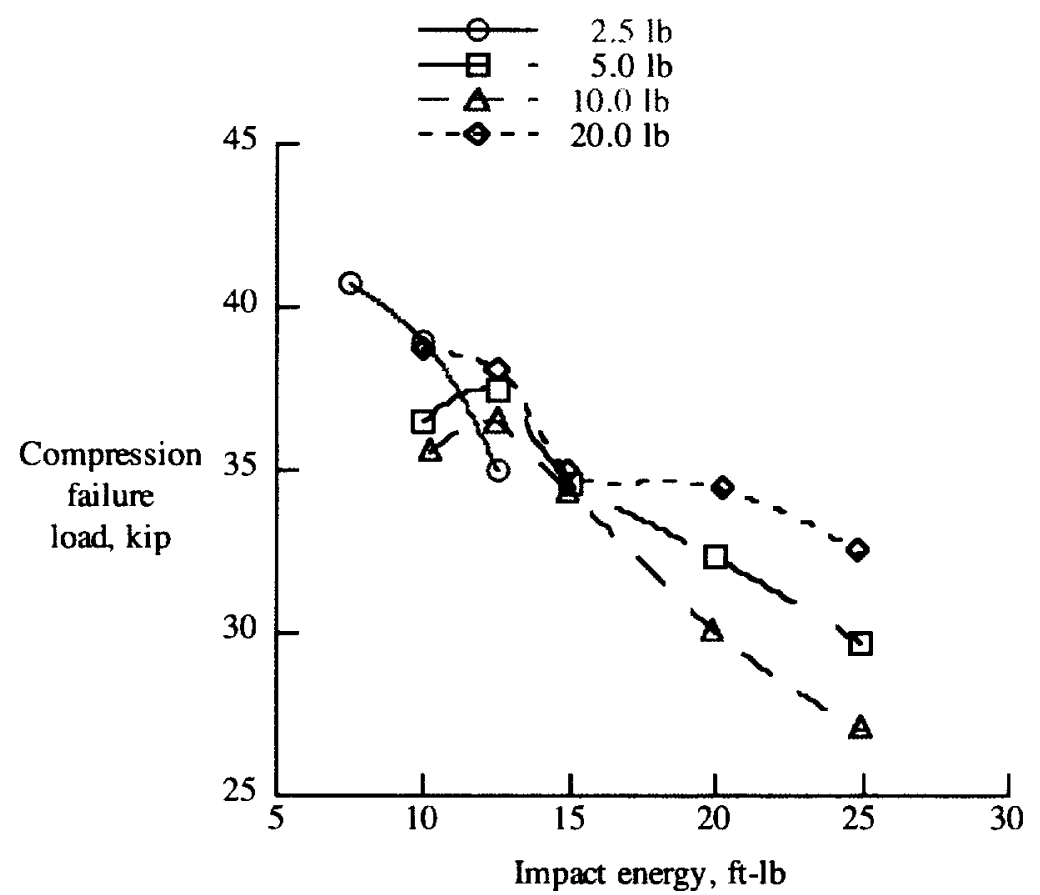

Figure 6. Compression-after-impact strength results for impacts with increasing energy levels and with different impactor weights.

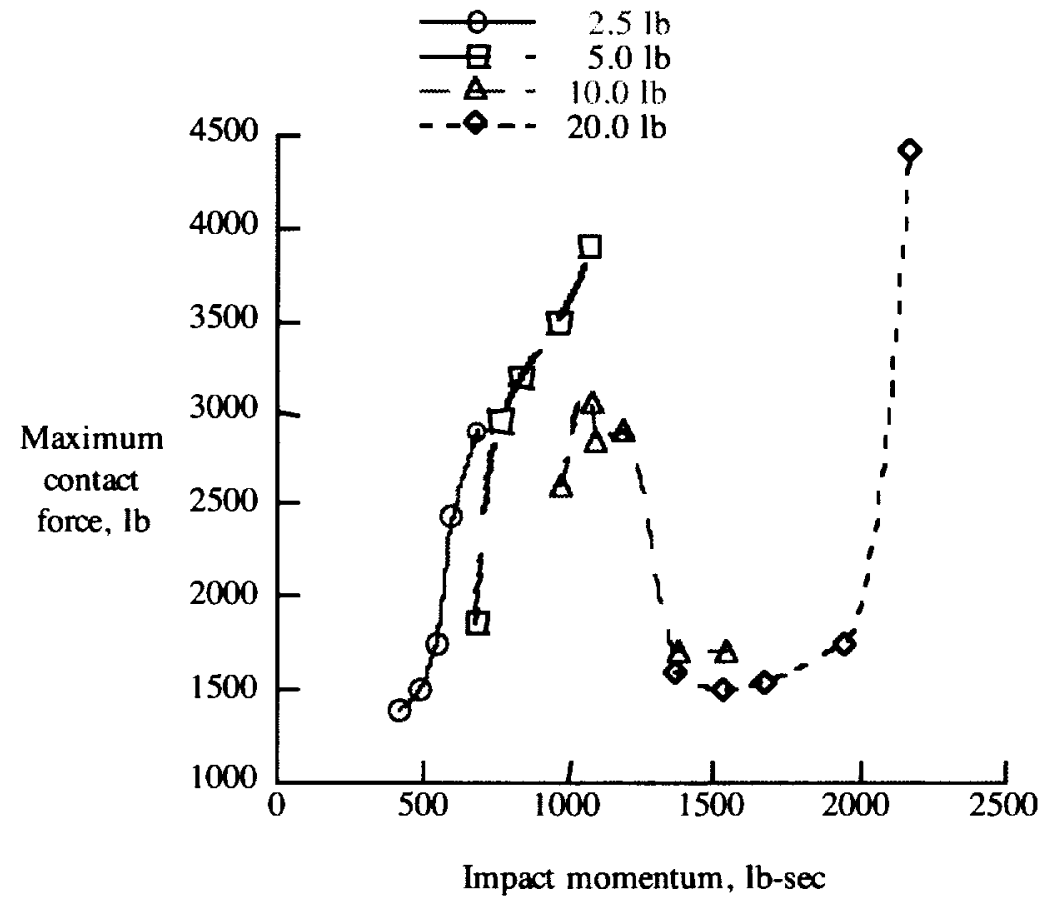

Figure 7. Experimental contact force results for impacts with increasing values of impactor momentum and with different impactor weights. 


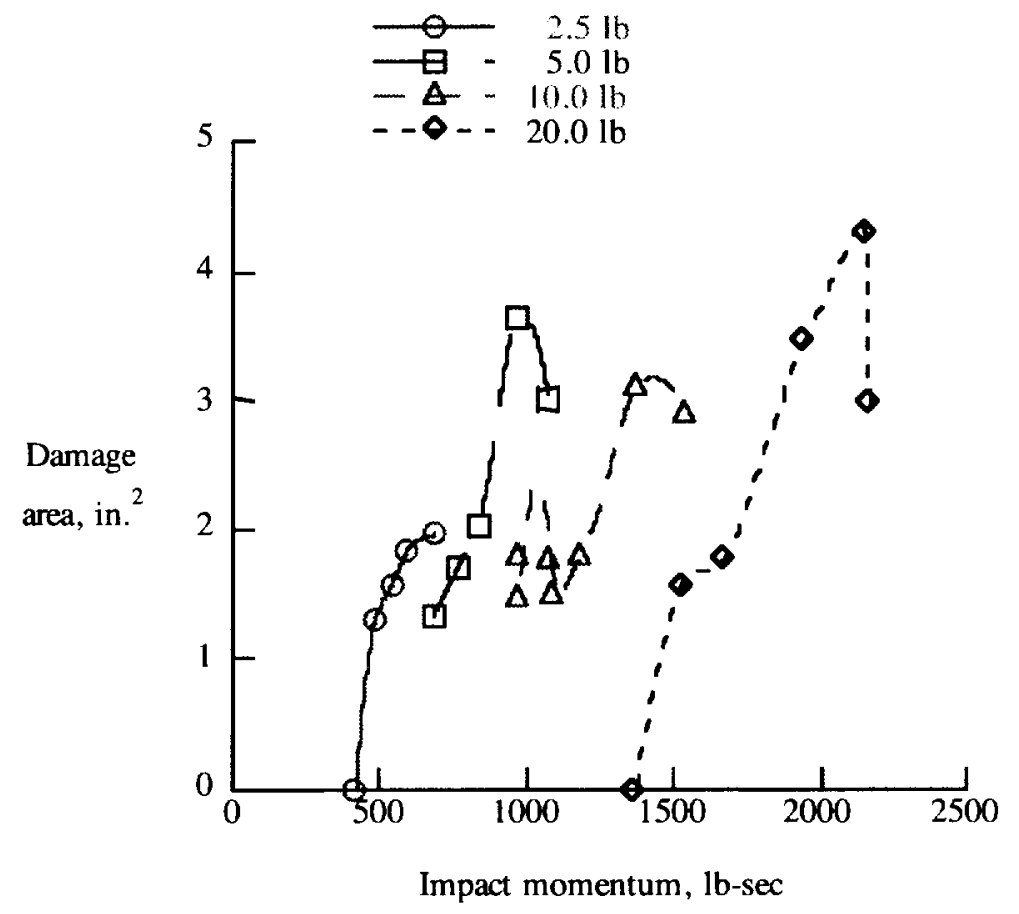

Figure 8. Experimental damage area results for impacts with increasing values of impactor momentum and with different impactor weights.

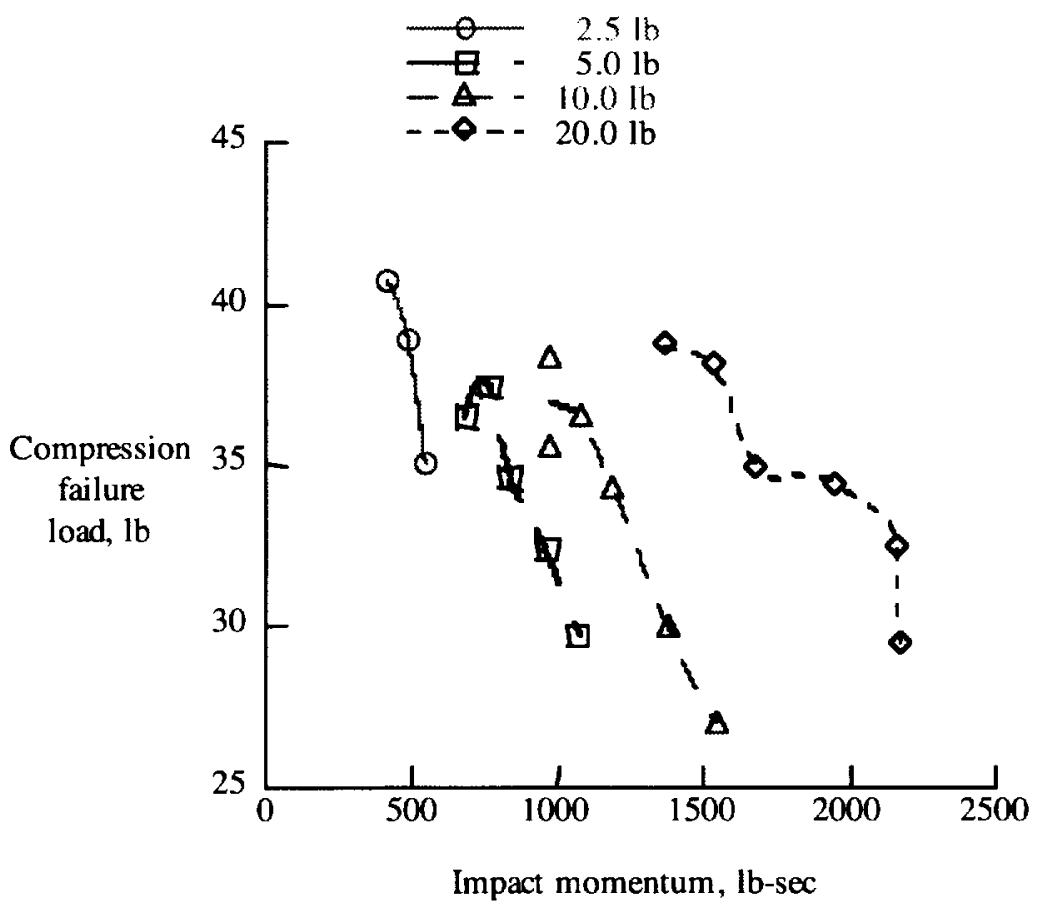

Figure 9. Compression-after-impact strength results for impacts with increasing values of impactor momentum and with different impactor weights. 
